\title{
Pancreatic Adenocarcinoma: Epidemiology, Role of EUS in Diagnosis, Role of ERCP, Endoscopic Palliation
}

\author{
Sameer Zar ${ }^{1}$, Darina Kohoutová ${ }^{1,2, *}$, Jan Bureš ${ }^{2}$
}

\section{ABSTRACT}

Pancreatic cancer is the seventh leading cause of cancer deaths worldwide and is associated with a poor survival rate. The vast majority of pancreatic cancers are inoperable at the time of diagnosis. In the absence of metastatic disease, operability depends on the extent of local disease; in particular, the presence or absence of vascular and lymph node involvement. Adequate staging is vital in deciding an appropriate treatment plan. Cross sectional imaging including CT, MRI and PET-CT are commonly used for staging. However, EUS is a useful adjunct for accurate loco-regional staging in addition to allowing diagnostic tissue samples to be obtained. Emerging EUS-guided therapeutic techniques have opened up new horizons in the management of pancreatic malignancy. EUS guidance can be used for coeliac plexus neurolysis in patients with intractable pain and fiducial placement in directing stereotactic radiotherapy. The majority of patients with cancer of the pancreatic head present with biliary obstruction. ERCP can be used to drain the obstructed biliary system with plastic or metal stents and offers an opportunity to confirm the diagnosis by obtaining brush cytology and forceps biopsy specimens. EUS-guided choledocho-duodenostomy or hepatico-gastrostomy is increasingly being employed for draining the biliary system if ERCP is unsuccessful.

\section{KEYWORDS}

pancreatic adenocarcinoma; endoscopic ultrasound; ERCP

AUTHOR AFFILIATIONS

${ }^{1}$ The Royal Marsden Hospital NHS Foundation Trust, Fulham Road, Chelsea, SW3 6JJ, London, United Kingdom

2 2nd Department of Internal Medicine - Gastroenterology, Charles University, Faculty of Medicine in Hradec Králové, University Hospital, Hradec Králové, Czech Republic

* Corresponding author: The Royal Marsden Hospital NHS Foundation Trust, Fulham Road, Chelsea, SW3 6JJ, London, United Kingdom; e-mail: Darina.Kohoutova@rmh.nhs.uk

Received: 2 August 2019

Accepted: 28 September 2019

Published online: 10 February 2020

Acta Medica (Hradec Králové) 2019; 62(4): 131-136

https://doi.org/10.14712/18059694.2020.1

(c) 2019 The Authors. This is an open-access article distributed under the terms of the Creative Commons Attribution License (http://creativecommons.org/licenses/by/4.0), which permits unrestricted use, distribution, and reproduction in any medium, provided the original author and source are credited. 


\section{PANCREATIC CANCER EPIDEMIOLOGY}

Pancreatic cancer is considered one of the deadliest cancers and is associated with a poor survival rate. Currently, it is the seventh leading cause of cancer deaths worldwide and the sixth leading cause in the UK (1). The incidence of pancreatic cancer is increasing, with 458,918 new cases diagnosed worldwide and 11,374 new cases in the UK according to the GLOBOCAN 2018 estimates (2).

While the causes of pancreatic cancer are still not completely known, non-modifiable and modifiable risk factors have been identified. Non-modifiable risk factors include familial cancer syndromes, increasing age, diabetes mellitus, and hereditary and other forms of chronic pancreatitis. Significant modifiable risk factors include smoking, obesity, toxins and dietary factors (such as non-vegetarian diet and alcohol) (3). There is also a higher incidence in developed countries and pancreatic cancer is slightly more common in men compared to women.

Pancreatic cancers can arise from the exocrine or neuroendocrine cells of the pancreas. Over $90 \%$ of pancreatic cancers are exocrine tumours, of which the vast majority are pancreatic ductal adenocarcinomas (PDACs). Pancreatic neuroendocrine tumours (NETs) make up less than $5 \%$ of all cases (4). All tumours are staged using various imaging modalities such as CT, MRI, PET-CT, endoscopic ultrasound (EUS) or laparoscopy. Adequate staging is vital in deciding appropriate treatment plan. The 8th edition of TNM (Tumour, Nodes, Metastases) staging system is currently used for pancreatic cancer, Table 1 (4).

Tab. 1 The 8th edition of TNM (Tumour, Nodes, Metastases) staging system of pancreatic cancer.

\begin{tabular}{|l|l|}
\hline T1 & Maximum tumor diameter $\leq 2 \mathrm{~cm}$ \\
\hline T2 & Maximum tumor diameter $>2 \mathrm{~cm}$ and $\leq 4 \mathrm{~cm}$ \\
\hline T3 & Maximum tumor diameter $>4 \mathrm{~cm}$ \\
\hline T4 & $\begin{array}{l}\text { Tumor involves the celiac axis, common hepatic artery } \\
\text { or the superior mesenteric artery }\end{array}$ \\
\hline N0 & No regional lymph node metastasis \\
\hline N1 & Metastasis in $1-3$ regional lymph nodes \\
\hline N2 & Metastasis in $\geq 4$ cm regional lymph nodes \\
\hline M0 & No distant metastasis \\
\hline M1 & Distant metastasis \\
\hline
\end{tabular}

The operability of pancreatic cancers depends on the extent of tumour spread locally and to distant organs as well as invasion of nearby vessels.

Patients with early-stage pancreatic cancer are usually clinically well. Symptoms of pancreatic cancer such as abdominal or mid-back pain, obstructive jaundice, and weight loss tend to occur after tumour invasion of surrounding tissue or metastatic spread (5). Consequently, patients tend to present late with advanced disease, with $79 \%$ of pancreatic cancer cases in England being diagnosed at Stage 3 or 4 and 21\% diagnosed at Stage 1 or 2. This likely contributes to the high mortality rates associated with pancreatic cancer. An analysis of 182 pancreatic cancer patients by Shigehiro et al. found that only 12 (6.6\%) patients survived more than 5 years after surgical resection (6).
According to 2010-11 data from Cancer Research UK, the 1-year, 5-year and 10-year survival rates of people diagnosed with pancreatic cancer in England and Wales are $21 \%, 3 \%$ and $1 \%$ respectively (1).

In England 2013-14, 10\% of patients diagnosed with pancreatic cancer had surgery to remove their primary tumour as part of their primary cancer treatment, including those who had received chemotherapy or radiotherapy. There is a clear link between disease stage at time of diagnosis and whether the patient received surgery, as illustrated in Table 2. Other factors that affect whether a patient receives surgery include the patient's generally health (i.e. comorbidities), age, and patient's own treatment preference (1).

Tab. 2 Pancreatic cancer: Percentage of patients receiving surgery to remove the tumour in the 9 months after diagnosis, male and female, all ages, England 2013-2014 (1).

\begin{tabular}{|l|l|}
\hline Stage at diagnosis & Percentage of patients \\
\hline All stages combined & $9.8 \%$ \\
\hline Stage 1 & $21.2 \%$ \\
\hline Stage 2 & $53.7 \%$ \\
\hline Stage 3 & $7.6 \%$ \\
\hline Stage 4 & $2.2 \%$ \\
\hline Unknown stage & $7.8 \%$ \\
\hline
\end{tabular}

\section{ROLE OF ENDOSCOPIC ULTRASOUND IN PANCREATIC CANCER}

Initial investigations in a patient with suspected pancreatic cancer include ultrasound scan of the abdomen, CT scan and MRI of the pancreas. However, over the last decade endoscopic ultrasound has emerged as an important modality in the diagnosis and management of pancreatic cancer. It not only augments the information obtained through other imaging modalities, but also offers the opportunity of obtaining tissue sample to confirm the diagnosis. In addition, it is emerging as a valuable therapeutic tool in the management of pancreatic cancer.

\subsection{EUS AND DIAGNOSTIC STAGING OF PANCREATIC CANCER}

Ultrasound is usually the initial diagnostic modality employed in patients who present with biliary obstruction. It can confirm the presence of biliary obstruction by detecting dilatation of intra-hepatic and extra-hepatic bile ducts, however, adequate visualisation of the lower common bile duct and pancreatic parenchyma is usually restricted due to the interference from gas in the gastrointestinal tract (7). A triple phase CT scan is the standard of care for the diagnosis of suspected pancreatic cancer. It is widely available and can detect the primary tumour, assess its local extent and relationship with the vasculature, identify spread to regional lymph nodes, and detect distant metastases (8). In patients who are unable to undergo contrast enhanced CT scanning due to allergy to iodine-based contrast agents, MRI provides an alternative 
imaging modality. It has higher sensitivity in identifying small liver metastases compared to CT scans (9). Positron Emission tomography (PET scan) can provide additional information in assessment of metastatic disease in some patients but has limited value in the assessment of the primary lesion (10).

These cross sectional imaging techniques are useful for accurate staging of the tumour, which is important in determining the treatment approach to the disease. Unfortunately, the vast majority of pancreatic cancers (approximately $85 \%$ ) are inoperable at the time of diagnosis due to the presence of metastatic disease or invasion of major vascular structures. In the absence of metastatic disease, operability depends on the extent of local disease; in particular, the presence or absence of vascular and lymph node involvement. The disease is frequently categorised into three groups, based on imaging results: operable, borderline operable, and locally advanced inoperable disease.

EUS provides high-resolution images of the pancreatic parenchyma as the EUS probe can be placed in close proximity of the lesion through the wall of the stomach or duodenum, depending on the location of the lesion. EUS can provide accurate loco-regional staging and can complement the findings of cross sectional imaging - especially in cases of borderline operable disease or in cases where CT and MRI are unable to detect a mass due to the enhancement pattern of the lesion (11).

EUS probes can either be radial or linear in configuration. Radial scopes provide images in a cross sectional plane similar to CT and MR imaging whereas linear probes provide imaging in a single plane parallel to tip of the scope (12). The endoscopist uses a 'station approach' to assess different parts of pancreas. The lesions in the pancreatic body and tail are evaluated from the gastric station whereas lesions in the pancreatic head and uncinate process are visualised through the duodenal stations. High-resolution images of the lesion are obtained using $7.5 \mathrm{MHz}$ frequency (range 5-20 MHz). This enables accurate T-staging of the tumour with a sensitivity and specificity comparable to modern cross sectional imaging (13). Most pancreatic cancers on EUS image appear as hypoechoic heterogenous lesions with irregular margins. The relationship of lesions to the coeliac trunk, portal vein confluence and superior mesenteric vein and artery is carefully assessed to determine operability of the lesion.

Ancillary techniques can be used to improve characterisation of the lesion with EUS, including elastography and contrast enhanced imaging. Elastography involves assessment of firmness or elasticity of a target lesion compared to the surrounding normal tissue. The elastography data can be displayed qualitatively as a colour overlay on the standard B mode image. Pancreatic cancers are firm lesions appearing as blue while inflammatory lesions appear as green-yellow and soft lesions as red. Therefore, elastography could be a helpful tool in a patient with chronic pancreatitis where the difference between inflammatory and cancerous components can be really difficult. Alternatively, a quantitative scale called strain ratio can be obtained by selecting a target area compared to a normal tissue at the same level, which is displayed as a numeric value. Contrast enhanced EUS involves using a specific intravenous ultrasound agent comprising of microbubbles, which can be detected flowing through the microvasculature of the lesion (14). The pancreatic cancers appear as hypochoic areas as they are usually hypovascular in nature (15). The role of these ancillary techniques in clinical practice remains investigational at present but experts believe that these can help in differentiating between different types of pancreatic lesions and enable targeted biopsies in difficult diagnostic situations.

Limited depth of penetration of the ultrasound waves prevents accurate assessment of structures that are located more than 5 or $6 \mathrm{~cm}$ from the EUS probe. EUS therefore has limited role, if any, in the assessment of distant lymph nodes or metastatic disease. In cases where the anatomy is distorted or surgically altered (e.g. the presence of biliary stents), it may not be possible to obtain optimal imaging. Similarly, in patients with chronic pancreatitis, presence of calcification can significantly limit the image quality (15).

The information obtained from EUS should be considered complimentary to other imaging modalities in establishing accurate staging of pancreatic cancer.

\subsection{EUS GUIDED TISSUE DIAGNOSIS}

The main advantage of EUS lies in the ability to obtain tissue samples for establishing the diagnosis. This has become possible through linear EUS scopes with large accessory channels. Specifically, designed needles are passed through the scope channel, which exit near the tip of the scope along the plane of linear EUS probe. Thus, allowing passage of the sampling needle through the wall of the stomach or duodenum under direct ultrasound guidance into the target lesion while avoiding any vascular structure. The sample can be either a cytology specimen obtained through a hollow needle or a fine core of tissue acquired through specially designed needles. The former is called fine needle aspiration (FNA) and latter fine needle biopsy (FNB). Cytology specimens are either directly smeared on the slides or placed in saline or other preservative solution for spinning in order to obtain tissue blocks. Biopsy cores on the other hand are placed into formalin for fixation. Pro-core biopsy provides histological tissue assessment, including cellular and connective tissue analysis, to obtain unequivocal evidence of invasive disease. It also allows immuno-histochemical staining, which can provide important information in confirming diagnosis $(11,16,17)$.

Tissue sampling is useful in cases where a diagnosis of pancreatic cancer is not clear on cross sectional imaging before embarking on major surgery. It is also considered mandatory by most institutions to obtain cytological or histological confirmation of malignancy in patients with borderline operable and inoperable tumours, prior to commencing neo-adjuvant or palliative chemotherapy respectively. In the era of targeted chemotherapy, it is envisaged that the need for tissue sampling is likely to increase and may be required at multiple points during the evolution of disease. 


\subsection{EUS GUIDED INTERVENTIONS IN PANCREATIC CANCER}

Emerging EUS-guided therapeutic techniques have opened up new horizons in the management of pancreato-biliary diseases. Patients with advanced pancreatic carcinoma with intractable pain unresponsive to analgesics, caused by infiltration of the coeliac plexus, can benefit from EUS-guided coeliac ganglion or coeliac plexus neurolysis. This involves EUS guided injection of absolute alcohol diluted with local anaesthetic agent bupivacaine either directly into coeliac ganglia or around the coeliac plexus nerves using dedicated needles (18).

EUS FNA needles have been used to place fiducials in the pancreatic tumour or local lymph nodes to guide stereotactic radiotherapy (19). Dedicated needles with pre-loaded fiducials are now available from some manufacturers and allow placement of 3 or 4 fiducials without the need to remove the needle assembly for reloading (20), thus greatly reducing the time required for the procedure. EUS allows precise placement of the fiducials under EUS guidance even in very small lesions (21).

EUS can also be used to drain the biliary system in patients when ERCP is unsuccessful due to an impassable biliary stricture, altered anatomy due to previous surgery or development of duodenal obstruction by tumour infiltration. EUS guided choledocho-duodenostomy involves puncturing the CBD above the stricture from the duodenal bulb with the EUS needle and inserting a fully covered metal biliary stent over a guide-wire under fluoroscopy (22). Alternatively, the biliary system can be drained after performing hepatico-gastrostomy by puncturing the dilated radicles of the left system in segment 2 or 3 of the liver. A guide-wire is then advanced into the CBD and across the stricture into the duodenum, allowing the anterograde placement of a stent, in a fashion similar to percutaneous trans-hepatic cholangiography (23). If the wire cannot be passed through the stricture, a specially designed stent can be placed across the gastric puncture site. The distal end of stent, which is uncovered, extends into the bile duct above the level of obstruction while the proximal covered end is left in the gastric lumen, allowing the retrograde drainage of bile. The covered gastric end of the stent prevents bile leaking into the peritoneum whereas the distal uncovered end helps anchoring the stent and allows drainage of side branches of the biliary system. Recent design change has incorporated a $70 \%$ covered portion on the gastric end $(24,26)$. These techniques require dilatation of the tract with a 5-6 Fr catheter, which can deliver electrocautery through its tip such as a cystgastrostomy needle. New stent designs have incorporated electrocautery rings on the tip of stent delivery system, which allows entire procedure to be completed with a single puncture under image guidance. More recently, lumen-apposing stents (LAMS) have been specifically designed to appose the lumen of the GI tract with another organ or cavity, such as dilated bile duct, pancreatic fluid collection or distended gallbladder. It can also be used to create a gastro-jejunostomy to bypass duodenal obstruction in advanced pancreatic malignancy (25).

Radiofrequency probes have been designed to induce thermal necrosis of focal pancreatic lesions such as small
NETs and pancreatic cancers in patients who are not surgical candidates due to co-morbidities. Investigators have looked at the option of delivering EUS-guided chemotherapeutic agents, immunoreactive drugs or oncolytic viruses directly into pancreatic tumours or inducing vascular necrosis by occluding feeding vessels to the tumour as treatment options (27).

\section{ERCP IN PANCREATIC CANCER}

Symptoms of biliary obstruction such as jaundice, pruritis, pale stools and dark urine are the presenting symptom complex in the majority of patients with lesions arising in the head of pancreas. ERCP offers both a diagnostic and therapeutic role in such patients. In contrast, patients with lesions arising in the body or tail of the pancreas usually do not present with biliary obstruction until they metastasise to the liver hilum. In these cases, biliary obstruction is a late symptom usually caused by obstruction from hilar lymph node enlargement or central metastases to the liver (28).

\subsection{THE DIAGNOSTIC ROLE OF ERCP IN PANCREATIC CANCER}

ERCP drainage of the biliary system provides an opportunity to confirm the diagnosis by a combination of biliary brush cytology and forceps biopsy specimens. These techniques yield a positive diagnostic sample in about one third of the patients (30). Additional techniques such as aspiration of bile, balloon dilatation of stricture or needle aspiration do not increase the diagnostic yield in a significant way. If a plastic stent was previously placed, cytological sample can be obtained from it. While forceps biopsies have a greater yield, they are more invasive and are associated with a greater risk of complications (i.e. bleeding, pancreatitis, perforation) compared to biliary brush cytology. Despite its low yield, ERCP tissue sampling plays a useful diagnostic role in patients who require biliary drainage at presentation. If ERCP does not yield a positive sample, diagnosis can be confirmed through EUS guided tissue acquisition, which has a significantly superior sensitivity (31).

\subsection{THE ROLE OF ERCP IN BILIARY DRAINAGE}

Many centres advocate proceeding to surgery without draining the biliary system due to the concern that it can potentially delay definitive surgery, particularly if the patient develops a complication such as pancreatitis, perforation or sepsis secondary to instrumentation of a blocked biliary system. Other centres favour pre-operative biliary drainage to reduce the risk of postoperative complications such as coagulopathy, sepsis and renal failure (33). Most agree that patients should undergo biliary drainage in the setting of biliary sepsis, severe symptoms such as intractable pruritis or if delay in surgery is contemplated. Patients with borderline operable tumours or those with advanced disease invariably require biliary drainage prior to commencing chemotherapy (29). 
The preferred method for biliary drainage is endoscopic stent placement as it is associated with fewer complications compared to percutaneous biliary drainage. The latter is generally reserved for cases where ERCP is unsuccessful or is not possible due to difficult anatomy (34). Biliary drainage can be achieved with either plastic or metal biliary stents. Although plastic stents are significantly cheaper, they tend to block within 10-12 weeks whereas metal biliary stents remain patent for longer $(35,36)$. Stent occlusion can cause sepsis requiring repeat procedures which can result in interruption of chemotherapy and delay in definitive surgical treatment (37).

In general, if the imaging favours a malignant process, a metal stent should be placed, as it does not interfere with

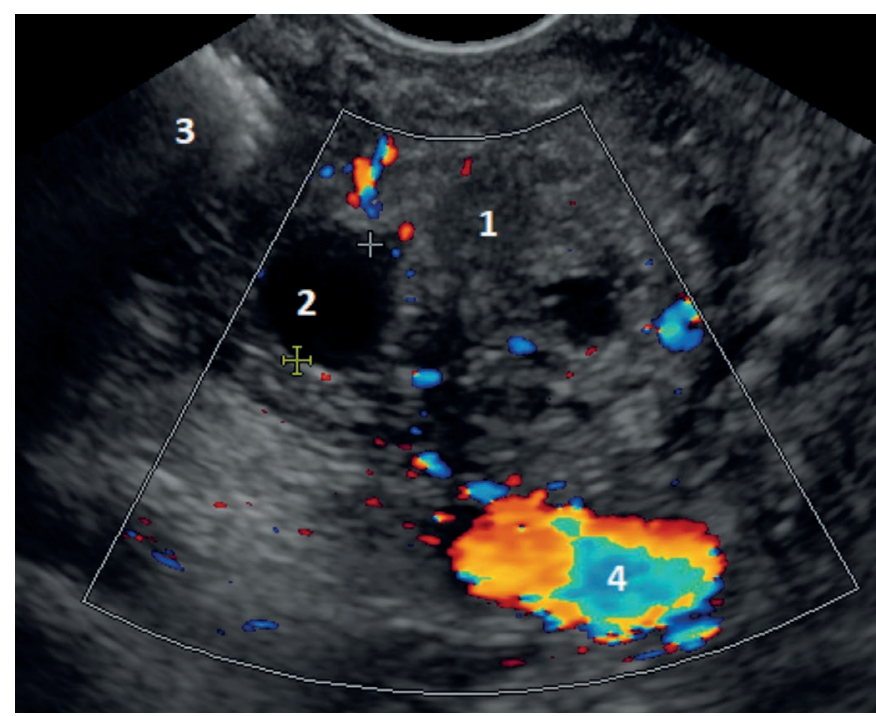

Fig. 1 Pancreatic adenocarcinoma of the uncinate process. 1 - carcinoma; 2 - dilated pancreatic duct; 3 - metal stent in the common bile duct (causing artephacts); 4 - superior mesenteric vein.

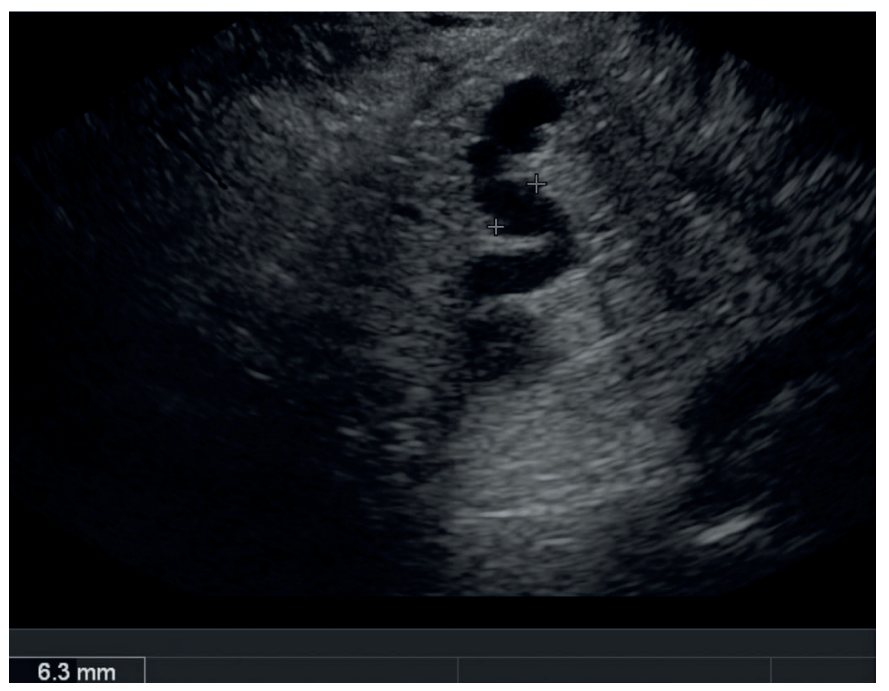

Fig. 3 Pancreatic adenocarcinoma of the uncinate process causing dilatation of the pancreatic duct to $6.3 \mathrm{~mm}$ in the neck of the pancreas. subsequent surgery, as long as the shortest possible stent is used. However, artefact from metal stents can impair visualisation during EUS and make it difficult to perform targeted needle aspiration especially in small lesions. In view of this, operators prefer performing EUS before stent insertion. However, this is not always possible due to limited availability of EUS as compared to ERCP, which is more widely practiced. Ideally, tumour staging and tissue acquisition by EUS should be followed immediately by either ERCP or EUS guided biliary drainage within the same session, if such a facility is available. If a diagnosis of malignancy is less certain, initial drainage of biliary obstruction with plastic stent is a reasonable option, which can be changed to a metal stent at a later date.

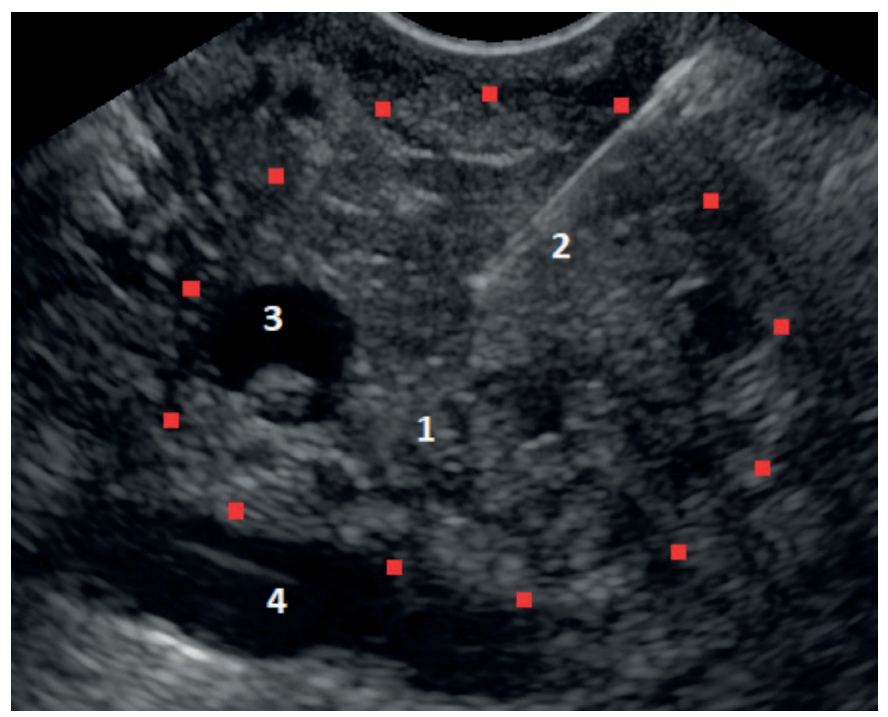

Fig. 2 Pancreatic adenocarcinoma of the uncinate process. 1 - carcinoma; 2 - FNB (fine needle biopsy) needle; 3 - dilated pancreatic duct with tissue inside; 4 - superior mesenteric vein.

\section{REFERENCES}

1. Cancer Research UK. Accessed in May 2019. https://www.cancer researchuk.org/health-professional/cancer-statistics/statistics-by -cancer-type/pancreatic-cancer

2. World Health Organisation. Cancer Today. International Agency for Research on Cancer. Accessed May 2019. http://gco.iarc.fr/today /home

3. Midha S, Chawla S, Garg PK. Modifiable and non-modifiable risk factors for pancreatic cancer: A review. Cancer Lett 2016;381(1): 269-77.

4. Cong L, Liu Q, Zhang R, et al. Tumor size classification of the 8th edition of TNM staging system is superior to that of the 7th edition in predicting the survival outcome of pancreatic cancer patients after radical resection and adjuvant chemotherapy. Sci Rep 2018; 8(1): 10383.

5. Vincent A, Herman J, Schulick R, Hruban RH, Goggins M. Pancreatic Cancer. Lancet 2011; 378(9791): 607-20.

6. Kure S, Kaneko T, Takeda S, Inoue S, Nakao A. Analysis of long-term survivors after surgical resection for invasive pancreatic cancer. HPB (Oxford) 2005; 7(2): 129-34.

7. Shrikhande SV, Barreto SG, Goel M, Arya S. Multimodality imaging of pancreatic ductal adenocarcinoma: a review of the literature. HPB 2012; 14(10): 658-68.

8. Fletcher JG, Wiersema MJ, Farrell MA, et al. Pancreatic malignancy: value of arterial, pancreatic, and hepatic phase imaging with multi-detector row CT. Radiology 2003; 229(1): 81-90.

9. Raman SP, Horton KM, Fishman EK. Multimodality imaging of pancreatic cancer-computed tomography, magnetic resonance imaging, and positron emission tomography. Cancer J 2012; 18(6): 511-22.

10. Delbeke D, Pinson CW. Pancreatic tumors: role of imaging in the diagnosis, staging, and treatment. J Hepatobiliary Pancreat Surg 2004; $11(1): 4-10$. 
11. Michl P, Pauls S, Gress TM. Evidence-based diagnosis and staging of pancreatic cancer. Best Pract Res Clin Gastroenterol 2006; 20(2): 227-51.

12. Gress F, Savides T, Cummings O, et al. Radial scanning and linear array endosonography for staging pancreatic cancer: a prospective randomized comparison. Gastrointest Endosc 1997; 45(2): 138-42.

13. DeWitt J, Devereaux B, Chriswell M, et al. Comparison of endoscopic ultrasonography and multidetector computed tomography for detecting and staging pancreatic cancer. Ann Intern Med 2004; 141(10): 753-63.

14. Sanchez MV, Varadarajulu S, Napoleon B. EUS contrast agents: what is available, how do they work, and are they effective? Gastrointest Endosc 2009; 69(2 Suppl): S71-7.

15. Fusaroli P, Spada A, Mancino MG, Caletti G. Contrast harmonic echo-endoscopic ultrasound improves accuracy in diagnosis of solid pancreatic masses. Clin Gastroenterol Hepatol 2010; 8(7): 629-34. e1-2.

16. Tierney WM, Adler DG, Chand B, et al. Echoendoscopes. Gastrointest Endosc 2007; 66(3): 435-42.

17. Iglesias Garcia J, Dominguez-Munoz JE. Endoscopic ultrasound-guided biopsy for the evaluation of pancreatic tumors. Gastroenterol Hepatol 2007; 30(10): 597-601.

18. Collins D, Penman I, Mishra G, Draganov P. EUS-guided celiac block and neurolysis. Endoscopy 2006; 38(9): 935-9.

19. Ammar T, Cote GA, Creach KM, Kohlmeier C, Parikh PJ, Azar RR. Fiducial placement for stereotactic radiation by using EUS: feasibility when using a marker compatible with a standard 22-gauge needle. Gastrointest Endosc 2010; 71(3): 630-3.

20. Park WG, Yan BM, Schellenberg D, et al. EUS-guided gold fiducial insertion for image-guided radiation therapy of pancreatic cancer: 50 successful cases without fluoroscopy. Gastrointest Endosc 2010; 71(3): 513-8.

21. Sanders MK, Moser AJ, Khalid A, et al. EUS-guided fiducial placement for stereotactic body radiotherapy in locally advanced and recurrent pancreatic cancer. Gastrointest Endosc 2010; 71(7): 1178-84.

22. Itoi T, Itokawa F, Tsuchiya T, Tsuji S, Tonozuka R. Endoscopic ultrasound-guided choledochoantrostomy as an alternative extrahepatic bile duct drainage method in pancreatic cancer with duodenal invasion. Dig Endosc 2013; 25(Suppl 2): 142-5.

23. Park SJ, Choi JH, Park DH, et al. Expanding indication: EUS-guided hepaticoduodenostomy for isolated right intrahepatic duct obstruction (with video). Gastrointest Endosc 2013; 78: 374-80.

24. Kahaleh M, Artifon EL, Perez-Miranda M, et al. Endoscopic ultrasonography guided biliary drainage: summary of consortium meet- ing, May 7th, 2011, Chicago. World J Gastroenterol 2013; 19(9): 1372-9.

25. Kunda R, Pérez-Miranda M, Will U, et al. EUS-guided choledochoduodenostomy for malignant distal biliary obstruction using a lumen-apposing fully covered metal stent after failed ERCP. Surg Endosc 2016; 30: 5002-8

26. Artifon EL, Aparicio D, Paione JB, et al. Biliary drainage in patients with unresectable, malignant obstruction where ERCP fails: endoscopic ultrasonography-guided choledochoduodenostomy versus percutaneous drainage. J Clin Gastroenterol 2012; 46(9): 768-74.

27. Wallace MB, Sabbagh LC. EUS 2008 Working Group document: evaluation of EUS-guided tumor ablation. Gastrointest Endosc 2009; 69(2 Suppl): S59-63.

28. Stapley S, Peters TJ, Neal RD, Rose PW, Walter FM, Hamilton W. The risk of pancreatic cancer in symptomatic patients in primary care: a large case-control study using electronic records. Br J Cancer 2012; 106(12): 1940-4.

29. Moss AC, Morris E, Leyden J, MacMathuna P. Malignant distal biliary obstruction: a systematic review and meta-analysis of endoscopic and surgical bypass results. Cancer Treat Rev 2007; 33(2): 213-21.

30. Adamsen S, Olsen M, Jendresen MB, Holck S, Glenthoj A. Endobiliary brush biopsy: intra- and interobserver variation in cytological evaluation of brushings from bile duct strictures. Scand J Gastroenterol 2006; 41(5): 597-603.

31. Singh H, Siddiqui AA. Endosonographic workup and preoperative biliary drainage for pancreatic cancer. Semin Oncol 2015; 42(1): 59-69.

32. Jailwala J, Fogel EL, Sherman S, et al. Triple-tissue sampling at ERCP in malignant biliary obstruction. Gastrointest Endosc 2000; 51(4 Pt 1): 383-90.

33. Kloek JJ, Heger M, van der Gaag NA, et al. Effect of preoperative biliary drainage on coagulation and fibrinolysis in severe obstructive cholestasis. J Clin Gastroenterol 2010; 44(9): 646-52.

34. Bonin EA, Baron TH. Preoperative biliary stents in pancreatic cancer. J Hepatobiliary Pancreat Sci 2011; 18(5): 621-9.

35. Saxena P, Kumbhari V, Zein ME, Khashab MA. Preoperative biliary drainage. Dig Endosc 2015; 27(2): 265-77.

36. Decker C, Christein JD, Phadnis MA, Wilcox CM, Varadarajulu S. Biliary metal stents are superior to plastic stents for preoperative biliary decompression in pancreatic cancer. Surg Endosc 2011; 25(7): 2364-7.

37. Boulay BR. Biliary stents for pancreas cancer with obstruction: the problem with plastic. J Gastrointest Oncol 2012; 3(4): 306-8. 